\title{
A modified procedure for parallel analysis of ordered categorical data
}

\author{
Ou Lydia LiU ANd Frank RiJMen \\ Educational Testing Service, Princeton, New Jersey
}

\begin{abstract}
Parallel analysis has been well documented to be an effective and accurate method for determining the number of factors to retain in exploratory factor analysis. The O'Connor (2000) procedure for parallel analysis has many benefits and is widely applied, yet it has a few shortcomings in dealing with missing data and ordinal variables. To address these technical issues, we adapted and modified the O'Connor procedure to provide an alternative method that better approximates the ordinal data by factoring in the frequency distributions of the variables (e.g., the number of response categories and the frequency of each response category per variable). The theoretical and practical differences between the modified procedure and the O'Connor procedure are discussed. The SAS syntax for implementing this modified procedure is also provided.
\end{abstract}

A critical component in the process of construct validation is to determine the number of factors on the basis of exploratory factor analysis (EFA). EFA is particularly useful when there is little theoretical basis for specifying either the number of factors or the relationship between the variables and latent factors (Hurley et al., 1997). The selection of the appropriate number of factors is one of the most important decisions in EFA, since underfactoring (selecting too few factors) or overfactoring (selecting too many factors) can have a deleterious impact on subsequent confirmatory analysis and item estimates (Brown, 2006). Ideally, the number of factors should represent qualitatively distinct constructs that conform to the underlying guiding theory.

Parallel analysis is one of the methods that helps one determine the number of factors in EFA (Carraher \& Buckley, 1995; Horn, 1965). The underlying rationale for parallel analysis is that the eigenvalues of the salient factors from real data with a valid latent factor structure should be larger than the eigenvalues of the corresponding factors that are generated from random data (Ford, MacCallum, \& Tait, 1986; Lautenschlager, 1989). Parallel analysis requires the construction of a number of correlation matrices of random variables based on the same sample size and number of variables found in the real data set. The mean eigenvalues from the random correlation matrices are compared to the eigenvalues from the real data correlation matrix. For example, the average of the first eigenvalues from the random data is compared to the first eigenvalue from the real data, and the average of the second eigenvalues from the random data is compared to the second eigenvalue from the real data. The eigenvalues observed from the real data should be larger than the corresponding average eigenvalues from the random data. Otherwise, the observed eigenvalues are considered to be consequences of sampling error (Glorfeld, 1995; Horn, 1965). Accordingly, a substantial and meaningful factor should account for more variance than one would expect from chance alone.

To perform a parallel analysis, a number of $k$ random data sets should be generated, and the average of the eigenvalues from these $k$ replications should be compared with the eigenvalues from the real data. There is no set rule regarding the number of random data sets that should be generated for parallel analysis. Horn (1965) suggested that the number should be reasonably large; other researchers have suggested from 500 to 1,000 repetitions (Hayton, Allen, \& Scarpello, 2004). However, no significant difference has been found between using one random data set or 100 random data sets (Crawford \& Koopman, 1979). Although there is no documented evidence, it appears that 50 data sets are reasonably enough for performing a parallel analysis (Hayton et al., 2004). In terms of evaluating the results, a modification has been proposed by Longman, Cota, Holden, and Fekken (1989) to Horn's approach of using the mean eigenvalues. The modified procedure for parallel analysis replaces the usual average of the eigenvalues with the upper 95 th percentile (or any other reasonable percentile) for determining the number of factors. This reduces the tendency to overextract or extract poorly supported factors.

Parallel analysis has been well documented to be a robust and accurate method for determining the number of factors to retain. Results from various studies have demonstrated that parallel analysis performed better than the widely used eigenvalue-greater-than-1.0 rule, the scree test, the maximum likelihood method, and the chi-square test (Bartlett, 1950; Eaton, Velicer, \& Fava, 1999; Humphreys \& Montanelli, 1975; Silverstein, 1987; Zwick \& Velicer, 1986).

Despite the proven advantage of parallel analysis, it is rarely used in the literature. A review by Hayton et al.

O. L.Liu, lliu@ets.org 
(2004) revealed that between 1990 and 1999, none of the 142 studies involving EFA in two major journals (the Academy of Management Journal and the Journal of Management) reported using parallel analysis. A major reason for the limited use of parallel analysis is the restricted access to software programs that can perform parallel analysis. As stated by Brown (2006, p. 29), "A practical drawback of the [parallel analysis] procedure is that it is not available in major statistical software packages such as SAS and SPSS."

\section{THE O'CONNOR PROCEDURE FOR CONDUCTING PARALLEL ANALYSIS}

O'Connor (2000) provides syntax for conducting parallel analysis in SPSS, SAS, and MATLAB. The Web site flash.lakeheadu.ca/ boconno $/$ nfactors.html provides syntax for programs suitable for data from normal distributions, as well as "raw" programs suitable for ordinal ${ }^{1}$ variables from nonnormal distributions. O'Connor uses a permutation of the raw data when dealing with ordinal data. Specifically, each column of the data matrix is permuted, so that the response vector of a new case consists of the responses of different old cases. This way, the dependence structure of the original data set is eliminated in the new data sets, but the marginal frequency distributions are preserved.

The O'Connor procedure does not permit missing data in the data matrix. It is assumed that users will deal with the missing data before they apply this program.

Since it is very common for ordinal data collected through psychological or educational surveys to contain missing values, it is important to provide a procedure that directly deals with missing data in parallel analysis. To meet this goal, we adapted the SAS syntax provided by O'Connor and made some modifications to provide an alternative way of conducting parallel analysis for ordinal data, allowing the presence of missing data.

\section{THE MODIFIED PROCEDURE FOR PARALLEL ANALYSIS}

In this section, we describe the innovative feature of the modified procedure, the major steps to implement this procedure, and a potential limitation of this procedure. Since this procedure is a modification of the O'Connor program, it is necessary to highlight the theoretical and practical differences between these two.

\section{Innovative Features of This Procedure}

On a theoretical level, instead of permuting the rows of the original data set, we proceed by estimating the relative frequencies for individual variables in the original data set and subsequently simulating the data for this variable in each new data set. We do this by drawing from a multinomial distribution for which the probabilities are given by these relative frequencies. Whereas, in O'Connor's permutation approach, dependencies between cases are introduced (i.e., the values for the last case are determined by the values of the other cases), all cases are independent draws in our approach, analogous to the parallel procedure for normal distributed variables. That is, our approach differs from the regular parallel analysis only in the distribution from which the cases are drawn (multinomial vs. normal distribution).

On a practical level, our procedure deals with random missing data in a more straightforward manner than does the O'Connor approach. For our approach, the frequencies for each variable are computed on all available cases. The simulated data will not contain missing values; a complete set of values is required by the subsequent SAS interactive matrix language code. In contrast, O'Connor's permutation-based approach does not deal with missing data directly. Of course, one could get around the missing data issue through, for example, imputing data for the missing observations. The most obvious way to do so would be to use the marginal relative frequencies of the variables, which is similar to our approach. Furthermore, imputing data will no longer ensure that the marginal frequencies of the simulated data exactly match those of the original data. Therefore, our approach has a practical advantage in terms of how it deals with random missing data.

\section{Implementing the Modified Procedure}

The procedure we developed, available in the SAS program, comprises three major steps. The first step imports data and generates a frequency table for the response categories. The second step generates $n$ random data sets from the multivariate distribution and computes eigenvalues from the generated data sets. Procedures for both principal component analysis (PCA) and factor analysis are made available. There has been a lack of agreement among experts on whether principal component eigenvalues or common and principal axis factor eigenvalues should be used to determine the number of factors. Some (e.g., Gorsuch, 1983; Humphreys \& Montanelli, 1975) have argued that if the eventual goal is to conduct a common factor analysis or principal axis factor analysis, then communalities should be placed on the diagonal of a correlation matrix before the eigenvalues are extracted based on the correlation matrix. Others (e.g., Cattell, 1966) have extracted and examined eigenvalues based on the correlation matrix of PCA and have used these eigenvalues to determine the number of factors for the common and principal axis factor analysis. The latter is the procedure used in the scree tests and in SPSS and SAS factor analysis. Here, in our SAS codes, we provide both options, allowing the user to decide whether to use PCA or factor analysis. The last step generates the mean and percentile of the eigenvalues for each component for the $n$ random data sets.

The SAS statements are provided in Appendixes A-C. ${ }^{2}$ Places where users should make changes according to their data characteristics are marked with a "*" that is followed by an explanation.

\section{A Potential Limitation of the Modified Procedure}

One limitation of this procedure is that it is currently only available in SAS software. Users who do not have access to SAS may not be able to use this program. However, 
the approach can easily be implemented in other packages, such as SPSS, C++, or FORTRAN, following the syntax provided here.

\section{CONCLUSION}

We hope that this modified procedure for parallel analysis can provide some practical value for determining the number of factors when conducting EFA. As described above, parallel analysis has been demonstrated to be an effective method for determining the number of factors to retain. The SAS procedure we developed offers a more straightforward solution for problems encountered when categorical data have missing cases. Readers are encouraged to use parallel analysis for factor retention when appropriate, in conjunction with content analysis and other types of evidence. The decision about factor structure should be made on the basis of consistent evidence from multiple sources that include both exploratory and confirmatory approaches.

\section{AUTHOR NOTE}

We thank Dan Eignor, Patrick Kyllonen, Richard Roberts, Yasuyo Sawaki, and Larry Stricker at ETS, and three anonymous reviewers for their helpful comments, suggestions, and edits on earlier drafts of this article. The authors also gratefully thank Kim Fryer, Jennifer Minsky, and Teresa Jackson for their editorial assistance. Correspondence concerning this article should be addressed to O. L. Liu, 666 Rosedale Road, Mailstop 16-R, Princeton, NJ 08541 (e-mail: 1liu@ets.org).

\section{REFERENCES}

BARTLETT, M. S. (1950). Tests of significance in factor analysis. British Journal of Mathematical \& Statistical Psychology, 3, 77-85.

BRown, T. A. (2006). Confirmatory factor analysis for applied research. New York: Guilford

CARraher, S. M., \& BuCKLey, M. R. (1995). The effect of retention rule on the number of components retained: The case of cognitive complexity and the PSQ. Proceedings of the Southern Management Association.

Cattell, R. B. (1966). The scree test for the number of factors. Multivariate Behavioral Research, 1, 245-276.

Crawford, C. B., \& Koopman, P. (1979). Inter-rater reliability of scree test and mean square ratio test of number of factors. Perceptual \& Motor Skills, 49, 223-226.
Eaton, C. A., Velicer, W. F., \& Fava, J. L. (1999). Determining the number of components: An evaluation of parallel analysis and the minimum average partial correlation procedures. Unpublished manuscript.

Ford, J. K., MacCallum, R. C., \& Tait, M. (1986). The application of exploratory factor analysis in applied psychology: A critical review and analysis. Personnel Psychology, 39, 291-314.

GLORFELD, L. W. (1995). An improvement on Horn's parallel analysis methodology for selecting the correct number of factors to retain. Educational \& Psychological Measurement, 55, 377-393.

Gorsuch, R. L. (1983). Factor analysis (2nd ed.). Hillsdale, NJ: Erlbaum.

Hayton, J. C., Allen, D. G., \& Scarpello, V. (2004). Factor retention decisions in exploratory factor analysis: A tutorial on parallel analysis. Organizational Research Methods, 7, 191-205.

HoRN, J. L. (1965). A rationale and test for the number of factors in factor analysis. Psychometrika, 30, 179-185.

Humphreys, L. G., \& Montanelli, R. G., JR. (1975). An investigation of the parallel analysis criterion for determining the number of common factors. Multivariate Behavioral Research, 10, 193-205.

Hurley, A. E., Scandura, T. A., Schriesheim, C. A., Brannick, M. T., Seers, A., VANDENBerg, R. J., ET AL. (1997). Exploratory and confirmatory factor analysis: Guidelines, issues, and alternatives. Journal of Organizational Behavior, 18, 667-683.

LautensChlager, G. J. (1989). A comparison of alternatives to conducting Monte Carlo analyses for determining parallel analysis criteria. Multivariate Behavioral Research, 24, 365-395.

Longman, R. S., Cota, A. A., Holden, R. R., \& Fekken, G. C. (1989). A regression equation for the parallel analysis criterion in principal components analysis: Mean and 95th percentile eigenvalues. Multivariate Behavioral Research, 24, 59-69.

O'CONNOR, B. P. (2000). SPSS and SAS programs for determining the number of components using parallel analysis and Velicer's MAP test. Behavior Research Methods, Instruments, \& Computers, 32, 396-402.

SiLVERSTEIN, A. B. (1987). Note on the parallel analysis criterion for determining the number of common factors or principal components. Psychological Reports, 61, 351-354.

ZWICK, W. R., \& VeLICER, W. F. (1986). Comparison of five rules for determining the number of components to retain. Psychological Bulletin, 99, 432-442.

\section{NOTES}

1. Ordinal data are categorical data in which there is a logical ordering to the categories.

2. Users can also contact the first author for the SAS code via e-mail at 1liu@ets.org. 


\section{APPENDIXA}

\section{Listing 1}

SAS Statements for Data Input

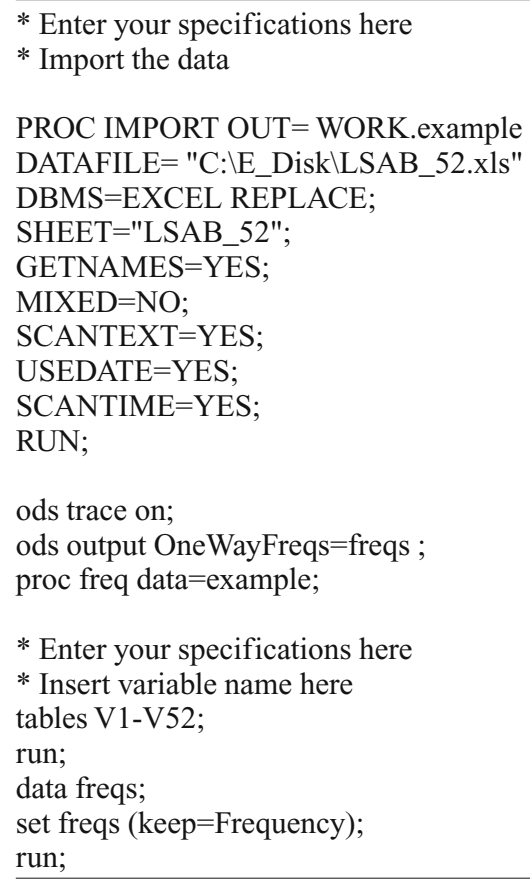

\section{APPENDIX B}

\section{Listing 2}

SAS Statements for Generating Variables From a Multinomial Distribution and Computing Eigenvalues

options nocenter nodate nonumber linesize $=90$;

title;

proc iml;

start RANDMULTINOMIAL( N, NumTrials, Prob);

$\mathrm{mP}=$ rowvec(Prob);

$\mathrm{d}=\operatorname{ncol}(\mathrm{mP})$;

if $\mathrm{N}<1$ then do;

print "The requested number of observations should be at least 1:" N; stop; end;

if NumTrials $<1$ then do;

print "The number of trials should be at least 1:" NumTrials; stop;

end;

if $\operatorname{abs}(1-\operatorname{sum}($ Prob $))>1 e-8$ then do;

print "The probabilities must sum to 1:" (sum(Prob))[label="Sum"]; stop;

end;

if $\operatorname{ncol}(\operatorname{loc}(\operatorname{Prob}>0))<\mathrm{d}$ then do;

print "Each probability should be positive:" Prob; stop;

end;

$\mathrm{b}=\mathrm{mP}$;

order $=\mathrm{d}+1-\operatorname{rank}(\mathrm{mP})$;

$\mathrm{mP}[$ order $]=\mathrm{b}$;

$X=j(n, d, 0)$;

$\mathrm{z}=0$;

do $\mathrm{i}=1$ to $\mathrm{N}$;

if $\mathrm{d}=1$ then do;

$\mathrm{X}[\mathrm{i}]=$ NumTrials; 
end;

else do;

$\mathrm{m}=$ NumTrials;

$\mathrm{q}=1$;

call randgen(z,'BINOM',m,mP[1]);

$\mathrm{X}[\mathrm{i}, 1]=\mathrm{z}$;

do $\mathrm{j}=2$ to $\mathrm{d}-1$ by 1 while $(\mathrm{m}>0)$;

$\mathrm{m}=\mathrm{m}-\mathrm{X}[\mathrm{i}, \mathrm{j}-1]$;

$\mathrm{q}=\mathrm{q}-\mathrm{mP}[\mathrm{j}-1]$

newp $=\mathrm{mP}[\mathrm{j}] / \mathrm{q}$;

call randgen(z,'BINOM',m,newp);

$\mathrm{X}[\mathrm{i}, \mathrm{j}]=\mathrm{z}$;

end;

$\mathrm{X}[\mathrm{i}, \mathrm{d}]=\mathrm{m}-\mathrm{z} ; / *$ to have the sum of $\mathrm{x} \_\mathrm{i}$ 's $=\mathrm{s} * /$

end;

end;

out $\mathrm{X}=\mathrm{X}$;

outX[ , 1:d ] = X[, order $]$;

return(outX);

finish;

store module=RANDMULTINOMIAL;

reset noname;

* Enter your specifications here

* The seed value can be changed by the user.

seed $=1953125$;

use freqs ;

read all into $\mathrm{F}$;

* Enter your specifications here

* Specify the number of cases (Ncases), number of variables (Nvars), number of randomly generated data sets (Ndatsets), and number of observed response category (Nrespcat) here. Note that the Nrespcat must be constant for all items.

Ncases $=238$;

Nvars $=52$

Ndatsets $=50$;

percent $=95$

Nrespcat $=4$;

* Enter your specifications here

* Specify the desired kind of parallel analysis, where $1=$ principal components analysis, and $2=$ principal axis/common factor analysis.

kind $=1$;

start setdiag(matname,vector);

do $\mathrm{i}=1$ to nrow(matname);

do $\mathrm{j}=1$ to ncol(matname);

if $(i=j)$ then; matname $[i, j]=\operatorname{vector}[i, 1]$;

end;end;

finish;

start rsum(matname);

rsums $=\mathrm{j}($ nrow $($ matname $), 1)$;

do rows $=1$ to nrow(matname);

dumr $=$ matname[rows, ;

rsums[rows, 1$]=$ sum(dumr);

end;

return(rsums);

finish;

if kind $=1$ then do;

evals $=\mathrm{j}($ Nvars,Ndatsets,-9999);

$\mathrm{nm} 1=1 /($ Ncases- 1$)$; 


\section{APPENDIX B (Continued)}

do nds $=1$ to Ndatsets;

$\mathrm{x}=\mathrm{j}(\mathrm{Ncases}, \mathrm{Nvars})$;

do a_var $=1$ to Nvars;

b $=(\text { a_var-1 })^{*}$ Nrespcat +1 ;

$\mathrm{e}=\mathrm{a} \_$var*Nrespcat;

Prob $=F[b: e, 1] / \operatorname{sum}(F[b: e, 1])$;

* Enter your specifications here

* The default number of categories for the random data is set to four. Change the ELSE IF command accordingly if the number of categories is some other number.

$\mathrm{y}=$ RANDMULTINOMIAL( Ncases, 1, Prob );

do a_case $=1$ to Ncases;

if $y[$ a_case, 1$]>0$ then

$\mathrm{x}$ [a_case,a_var] $=1$;

else if $y[$ a_case, 2$]>0$ then

$x[$ a_case,a_var $]=2$;

else if $y\left[a \_c a s e, 3\right]>0$ then

$\mathrm{x}\left[\mathrm{a} \_\right.$case,a_var] $=3$;

else if $y\left[a \_c a s e, 4\right]>0$ then

$\mathrm{x}\left[\mathrm{a} \_\right.$case,a_var $]=4$;

end;

end;

$\mathrm{vcv}=\mathrm{nm} 1 *(\mathrm{t}(\mathrm{x}) * \mathrm{x}-((\mathrm{t}(\mathrm{x}[+]), * \mathrm{x}[+]$,$) / Ncases ))$

$\mathrm{d}=\operatorname{inv}(\operatorname{diag}(\operatorname{sqrt}(\operatorname{vecdiag}(\mathrm{vcv}))))$;

evals[,nds] $=\operatorname{eigval}(\mathrm{d} * \mathrm{vcv} * \mathrm{~d})$;

end;

end;

if kind $=2$ then do;

evals $=\mathrm{j}($ Nvars,Ndatsets,-9999);

$\mathrm{nm} 1=1 /($ Ncases-1);

do nds $=1$ to Ndatsets;

$\mathrm{x}=\mathrm{j}($ Ncases,Nvars);

do a_var $=1$ to Nvars;

$\mathrm{b}=($ a_var-1)*Nrespcat +1 ;

$\mathrm{e}=\mathrm{a} \_$var*Nrespcat;

Prob $=F[b: e, 1] / \operatorname{sum}(F[b: e, 1])$;

$\mathrm{y}=$ RANDMULTINOMIAL( Ncases, 1, Prob );

do a_case $=1$ to Ncases;

if $y[$ a_case, 1$]>0$ then

$\mathrm{x}\left[\mathrm{a} \_\right.$case,a_var] $=1$;

else if y[a_case, 2] $>0$ then

$\mathrm{x}\left[\mathrm{a} \_\right.$case,a_var] $=2$;

else if $y[$ a_case, 3$]>0$ then

$\mathrm{x}[$ a_case,a_var] $=3$;

$\mathrm{x}\left[\mathrm{a} \_\right.$case,, $\mathrm{a} \_$var $]=4$;

end;

end;

$\mathrm{vcv}=\mathrm{nm} 1 *(\mathrm{t}(\mathrm{x}) * \mathrm{x}-((\mathrm{t}(\mathrm{x}[+]), * \mathrm{x}[+]$,$) / Ncases )) ;$

$\mathrm{d}=\operatorname{inv}(\operatorname{diag}(\operatorname{sqrt}(\operatorname{vecdiag}(\mathrm{vcv}))))$;

$\mathrm{r}=\mathrm{d} * \mathrm{vev} * \mathrm{~d}$;

$\operatorname{smc}=1-(1 / \operatorname{vecdiag}(\operatorname{inv}(r)))$;

run setdiag $(\mathrm{r}, \mathrm{smc})$;

evals[,nds] = eigval(r);

end;

end;

num $=$ round $(($ percent $*$ Ndatsets $) / 100)$;

results $=\mathrm{j}($ nvars $, 3,-9999)$;

$\mathrm{s}=1:$ Nvars; 
results $[, 1]=\mathrm{t}(\mathrm{s})$;

do $\operatorname{root}=1$ to Nvars;

$\operatorname{ranks}=\operatorname{rank}($ evals$[$ root, $])$;

do $\mathrm{col}=1$ to Ndatsets;

if $(\operatorname{ranks}[1, \mathrm{col}]=$ num $)$ then do;

results[root,3] $=$ evals [root,col];

$\mathrm{col}=$ Ndatsets;

end;

end;

end;

results $[, 2]=$ evals $[,+]$ / Ndatsets;

\section{APPENDIX C}

Listing 3

SAS Statements for Output Options

print, "Parallel Analysis:";

if $($ kind $=1)$ then; print, "Principal Components";

if $($ kind $=2)$ then do;

print "Principal Axis / Common Factor Analysis";

print "Compare the random data eigenvalues below to the";

print "real-data eigenvalues that are obtained from a";

print "Common Factor Analysis in which the \# of factors";

print "extracted equals the \# of variables/items, and the";

print "number of iterations is fixed at zero (maxiter $=0$ ), as in:";

print "proc factor data=trial priors $=$ smc maxiter $=0$; run; ";

print "Or use the 'rawpar.sas program' to obtain the ";

print "baseline real-data eigenvalues.";

end;

specifs $=($ ncases $/ /$ Nvars // Ndatsets // percent $)$

rlabels $=\{$ "Ncases" "Nvars" "Ndatsets" "Percent" $\}$;

print/ "Specifications for this Run:", specifs[rowname=rlabels];

clabels=\{"Root" "Means" "Prcntyle" $\}$;

print "Random Data Eigenvalues", results[colname=clabels format=12.6];

quit;

(Manuscript received July 26, 2007;

revision accepted for publication December 1, 2007.) 